\title{
Nonlinear refractive index of ultrafast laser inscribed waveguides in gallium lanthanum sulphide
}

\author{
Giorgos Demetriou, ${ }^{1}$ Daniel W. Hewak, Andrea Ravagli, Chris Craig, ${ }^{2}$ \\ AJOY KAR ${ }^{1, *}$ \\ ${ }^{1}$ Institute of Photonics and Quantum Sciences, School of Engineering and Physical Sciences, David Brewster Building, Heriot-Watt University, \\ Edinburgh, EH11 4AS, United Kingdom \\ ${ }^{2}$ Optoelectronics Research Centre, University of Southampton, Southampton SO40 4UG, UK \\ *Corresponding author: $\underline{\text { a.k.kar@hw.ac.uk }}$
}

Received XX Month XXXX; revised XX Month, XXXX; accepted XX Month XXXX; posted XX Month XXXX (Doc. ID XXXXX); published XX Month XXXX

\begin{abstract}
We demonstrate ultrafast all-optical switching in femtosecond laser inscribed nonlinear directional couplers in gallium lanthanum sulphide operated at $1.55 \mu \mathrm{m}$. We report on the evaluation of the nonlinear refractive index of the waveguides forming the directional couplers by making use of the switching parameters. The nonlinear refractive index is reduced by the inscription process to about 4-5 times compared to bulk material. (C) 2015 0ptical Society of America
\end{abstract}

OCIS codes: (220.4610) Optical fabrication; (130.2755) Glass waveguides; (230.1150) All-optical devices; (190.3270) Kerr effect; (190.7110)

Ultrafast nonlinear optics; (160.4330) Nonlinear optical materials.

http://dx.doi.org/10.1364/OL.99.099999

\section{INTRODUCTION}

Despite the continuing progress in electronics technology, there is a growing interest in the development of optical switching and processing devices due to their potential of operating at speeds and bandwidths much greater than those achievable with electronic devices. This rapid development of optical communications requires novel materials with large and ultrafast nonlinear optical responses in the femtosecond or picosecond domain for fabricating compact, lowthreshold all-optical switching (AOS) and processing devices.

Chalcogenide glasses (ChGs) are a particularly appealing candidate for such devices as they present high third-order (Kerr) nonlinearities (up to a thousand times that of silica) [1], low multi-photon absorption [2] and high photosensitivity [1], properties which allow for switching devices with ultrafast response, shorter interaction lengths, lower switching threshold and higher figures of merit.

Of all the ChGs, gallium lanthanum sulphide (GLS) is a particularly attractive one and a much-awaited alternative to toxic arsenic-based glasses. It presents optical transparency from the visible wavelengths extending in the infrared up to $10 \mu \mathrm{m}$ and thermal stability up to $550^{\circ} \mathrm{C}$ $[3,4]$. In addition, GLS can be melted in a large scale without the requirement of a sealed ampoule environment making production and processing safer, easier and more economical [4].

In this work, we have utilized the ultrafast laser inscription (ULI) technique [5, 6] to fabricate optical directional couplers in polished GLS glass substrates. ULI is a novel fabrication technique which offers the promise of highly functional optical circuits in three-dimensional geometries $[7,8]$ that are not possible with commercial optical fiber or planar lightwave circuit (PLC) fabrication methods. It relies on the nonlinear absorption of sub-bandgap photons to induce permanent structural changes to a material. These changes can manifest themselves in multiple ways, including a change in refractive index, which can be exploited to confine and guide light in ULI fabricated structures in bulk substrates $[9,10]$.

An evaluation of the linear refractive index profiles of ULI fabricated waveguides in GLS substrates was performed in a previous work [11] by our group. Here we expand our studies. We investigate the effect the inscription process has on the nonlinear refractive indices of the fs-laser inscribed directional couplers in GLS. We use a femtosecond OPA and the closed-aperture Z-scan technique [12] to measure the magnitude of the nonlinear refractive index coefficient, $n_{2}$, of the bulk substrate. We then evaluate the nonlinear refractive index of the waveguides forming the directional couplers by making use of the switching parameters when the directional couplers are operated as all-optical switches and compare with bulk GLS.

All-optical switching devices which exploit a nonlinear refractive change have attracted much attention because of their ultrafast response. In this approach the irradiance-dependent refractive index, $n=n_{0}+n_{2} I$, is used to demonstrate all-optical switching in the nonlinear medium. Here, $n_{o}$ is the linear component of the refractive index, $n_{2}$ is the nonlinear refractive index coefficient and $I$ is the irradiance. A nonlinear directional coupler, intended to operate as an all-optical switch, is designed in such way that when a low irradiance input is applied, a maximum amount of cross coupling is observed. Then, as the input irradiance is increased, the refractive index of the 
bar waveguide, following the above equation, increases, detuning the coupling coefficient between the two adjacent waveguides and causing most of the light to remain in the bar port in the output; therefore, achieving all-optical switching.

\section{DEVICE FABRICATION}

All structures reported in this letter were fabricated using a modelocked Yb-doped fiber laser emitting $360 \mathrm{fs}$ pulses at a central wavelength of $1045 \mathrm{~nm}$ and a pulse repetition rate of $500 \mathrm{kHz}$. The substrates were mounted on air bearing Aerotech stages and the pulses from the fabrication laser were focused inside the substrates to a depth of $240 \mu \mathrm{m}$ from the top surface using a 0.4 NA aspheric lens. The pulse energy incident on the samples was chosen to be $58 \mathrm{~nJ}$, whereas the translation speed was set to $12 \mathrm{~mm} / \mathrm{s}$. The substrate translation was perpendicular to the laser beam direction. The cross section of the inscribed waveguides forming the directional couplers was controlled using the well-known multiscan waveguide shaping technique $[13,14]$, yielding waveguides with rectangular crosssections. For each of the couplers, 10 scans with a scan-to-scan separation of $0.36 \mu \mathrm{m}$ were used. The above are the optimal ULI parameters for realising high quality single-mode waveguides at $1550 \mathrm{~nm}$ and were identified in a previous work by our group [11]. After fabrication, the input and output facets of the substrates were polished to optical quality.

All the directional couplers used in this work had the same geometric layout (Fig. 1). They were formed from two waveguides, single-mode at $1550 \mathrm{~nm}$. Each of the waveguides consisted of straight input and output sections linked to s-bends leading to a straight interaction region of length $\mathrm{L}$. The input and output ports of the two waveguides forming each coupler were separated by more than 100 $\mu \mathrm{m}$ to avoid evanescent coupling in any other sections of the coupler than the interaction length. This was achieved by choosing the lateral displacement in the $\mathrm{x}$ axis (x-offset) to be $50 \mu \mathrm{m}$ for each of the waveguides. The bend regions of the waveguides were inscribed by choosing a displacement of $5 \mathrm{~mm}$ along the propagation direction ( $\mathrm{y}$ offset) and a radius of curvature of $110 \mu \mathrm{m}$, resulting in waveguides which presented zero bend losses. The zero bend losses were verified by observing that waveguides of the above outlined geometry exhibited the same total throughput as a straight single-mode, at $1550 \mathrm{~nm}$, waveguide of the same length inscribed with the same laser parameters. The propagation losses in straight ultrafast laser inscribed waveguides in GLS were previously found to be in the order of $\sim 0.8 \mathrm{~dB}$ $\cdot \mathrm{cm}^{-1}[15]$.

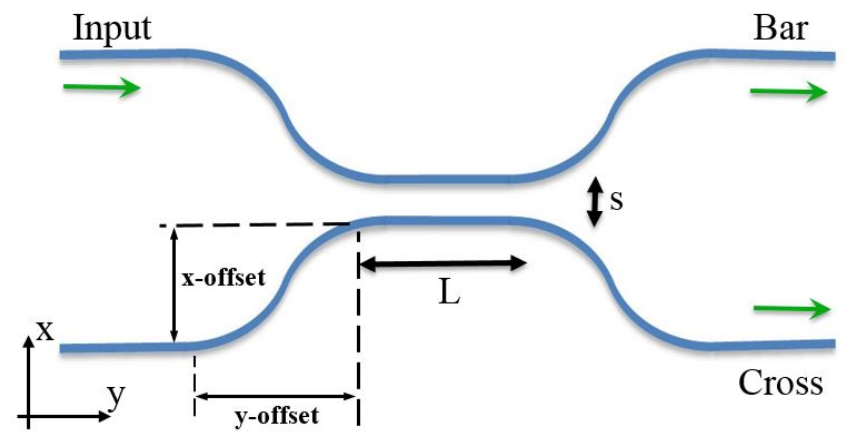

Fig. 1. Schematic of the directional coupler structure, where $s$ is the core-to-core separation between the waveguides along the interaction length (L).

While keeping all other parameters constant, a series of directional couplers were inscribed using interaction lengths between 3.55 and
$8.25 \mathrm{~mm}$ in steps of $50 \mu \mathrm{m}$. For all couplers, the core-to-core separation in the interaction region was set to $7.5 \mu \mathrm{m}$ from the center of the cores.

\section{ALL-OPTICAL SWITCHING}

As discussed earlier, a directional coupler intended to be operated as an all-optical switch is designed such way that for a low irradiance input a maximum amount cross coupling in the output is observed. Therefore, prior to carrying out the switching experiment, the output relative transmission for the bar and cross ports for each of the couplers was measured by coupling light from a $1550 \mathrm{~nm}$ CW fiber laser to the input port. The use of the CW laser ensured that no nonlinearities were excited whilst propagating light in the directional couplers, verifying the output cross-to-bar ratio of the couplers at low irradiances before any switching occurs.

A single beam experiment was then performed to examine the operation of the couplers as all-optical switches. A regeneratively amplified Ti:Sapphire source delivering 100 fs pulses, pumping an optical parametric amplifier (Newport Spectra-Physics OPA 800) at a repetition rate of $1 \mathrm{kHz}$ was used as input. The output from the OPA was centered at $1550 \mathrm{~nm}$ and after being passed through a diamond pinhole held in vacuum, ensuring its Gaussian shape, was coupled into and collected from the directional couplers using two AR coated 16X (0.25NA) aspheric lenses. The input irradiance was measured before the sample using a pyro-electric power meter (Laser Probe Inc. RkP$575)$, whereas the output light from the couplers was collected using a large-area germanium photoreceiver (New Focus 2033). The output cross-to-bar ratio as a function of the input irradiance for two directional couplers inscribed with different interaction lengths while keeping all the other parameters constant is shown in figure 2 .
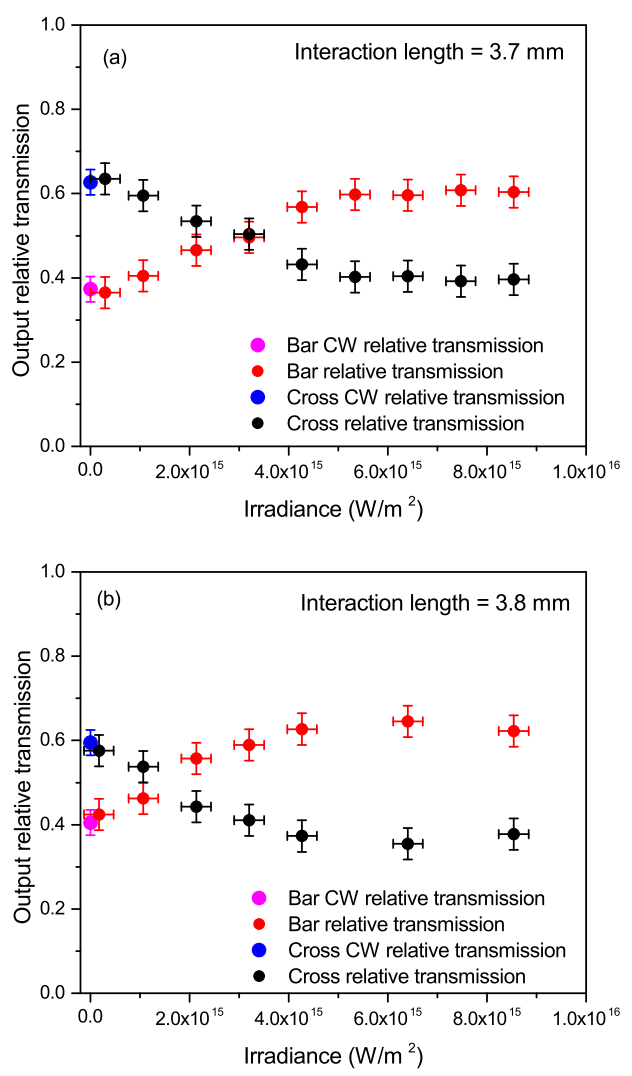

Fig. 2. Output relative transmission for the bar (red) and cross (black) ports as a function of the input irradiance for (a) a directional coupler with an interaction length of $3.7 \mathrm{~mm}$ and (b) a directional coupler with 
an interaction length of $3.8 \mathrm{~mm}$. The pink and blue circles in both graphs represent the bar and cross output ratio in the linear regime when using a $1550 \mathrm{~nm}$ CW fiber laser as input.

Coupler 1 was chosen to have an interaction length of $3.7 \mathrm{~mm}$, whereas for coupler 2 the interaction length was set to $3.8 \mathrm{~mm}$. The cross-to-bar output ratios, recorded with the $1550 \mathrm{~nm}$ CW laser as an input prior the switching experiment was performed, are shown in the same axes system (at zero irradiance) with pink and blue circles.

As can be seen from the figure, for a low irradiance input about $60 \%$ of the light is in the cross port at the output, whereas as the irradiance increases the bar-to-cross output ratio changes until it is completely reversed to about 60-40 \% bar-to-cross at the highest irradiances used in this experiment. The incident irradiance required for coupler 1 (Fig. 2a) to completely switch from the cross to the bar state was about $5.34 \times 10^{15} \mathrm{~W} / \mathrm{m}^{2}$, whereas the incident irradiance required for the second coupler (Fig. 2b) to completely switch from the cross to the bar state was about $4.27 \times 10^{15} \mathrm{~W} / \mathrm{m}^{2}$. The lower irradiance required to switch coupler 2 is consistent with its longer interaction length outlining the common dilemma of the trade-off between power consumption and device dimensions when designing all-optical processing devices.

\section{EVALUATION OF THE NONLINEAR REFRACTIVE INDEX FROM THE SWITCHING PARAMETERS}

The phase change $\Delta \Phi$ due to nonlinear refraction is [16]:

$$
\Delta \Phi=\frac{2 \pi L n_{2} I}{\lambda}
$$

where $L$ in this case is the interaction length of the directional couplers, $\lambda$ is the operating wavelength and I the is the irradiance.

The phase change, $\Delta \Phi$, required to switch a nonlinear directional coupler is in the order of $4 \pi$ [17]. Using this along with the corresponding interaction length and switching irradiance for each of the couplers of figure 2 in equation 1 , and after accounting for the losses due to Fresnel reflection at the input facet, the $\mathrm{n}_{2}$ of the modified region is calculated. The yielded $\mathrm{n}_{2}$ for coupler 1 was $1.89 \times 10^{-19} \mathrm{~m}^{2} / \mathrm{W}$, whereas the yielded value for coupler 2 was $2.30 \times 10^{-19} \mathrm{~m}^{2} / \mathrm{W}$.

In a similar work demonstrating all-optical switching in an aluminum gallium arsenide (AlGaAs) directional coupler [18] the critical irradiance, $\mathrm{I}_{c}$, was identified as the irradiance required to switch a half beat-length directional coupler to the condition where equal output powers emerge from the bar and cross waveguides. This critical irradiance was given as:

$$
I_{c}=\frac{\lambda}{L n_{2}}
$$

Adapting to our case, the critical irradiance for coupler 1 is $3.20 \times 10^{15}$ $\mathrm{W} / \mathrm{m}^{2}$, whereas the critical irradiance for coupler 2 is $1.49 \times 10^{15} \mathrm{~W} / \mathrm{m}^{2}$. Using these critical irradiances, again after accounting for the losses due to Fresnel reflection at the input, along with the interaction lengths for each of the couplers in the above equation the yielded $\mathrm{n}_{2}$ values are $1.58 \times 10^{-19} \mathrm{~m}^{2} / \mathrm{W}$ and $3.29 \times 10^{-19} \mathrm{~m}^{2} / \mathrm{W}$ for couplers 1 and 2 respectively, in good agreement with the values calculated with equation 1.

The nonlinear refractive index coefficient of bulk GLS at $1550 \mathrm{~nm}$ was measured via the closed-aperture Z-scan technique. The output of the OPA outlined above was focused using a $200 \mathrm{~mm}$ focal length calcium fluoride lens after being passed through a diamond pinhole held in vacuum to ensure its Gaussian shape. A $1 \mathrm{~mm}$ thick GLS sample was then translated through the focus along the beam path whilst recording the far field transmittance through an aperture using a largearea germanium photoreceiver (New Focus 2033). Figure 3 shows a closed-aperture Z-scan trace performed at a pulse energy of $100 \mathrm{~nJ}$ with an aperture transmission of $50 \%$. The theoretical fit uses the simplified closed aperture fitting formula from Sheik-Bahae et al. [12]. The theoretical fit corresponds to a nonlinear refractive index coefficient of $(9.7 \pm 0.94) \times 10^{-19} \mathrm{~m}^{2} / \mathrm{W}$.

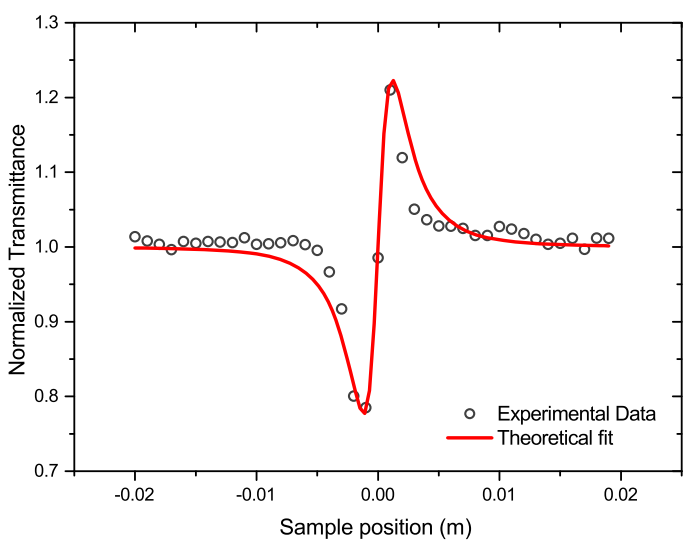

Fig. 3. Closed-aperture Z-scan trace of GLS at $1550 \mathrm{~nm}$ performed at a pulse energy of $100 \mathrm{~nJ}$. The theoretical fit (solid red line) is calculated using an $\mathrm{n}_{2}$ coefficient of $9.7 \times 10^{-19} \mathrm{~m}^{2} / \mathrm{W}$.

This value is about 4-5 times higher than the ones calculated for the modified area of the directional couplers by using the nonlinear phase change. By considering the values obtained using the critical irradiances, the $\mathrm{n}_{2}$ values of the modified area are calculated to be 3-6 times lower than the one measured for the bulk. These findings indicate that the nonlinear refractive index of GLS is reduced by the inscription process.

\section{DISCUSSION}

This trend of decreased nonlinearity for the modified area was also observed in straight ULI GLS waveguides [10], in a work performed from previous members of our group. In that work the authors derived the nonlinear phase change from the self-phase modulation (SPM) peaks and calculated the $\mathrm{n}_{2}$ coefficient of the modified area using equation 1 . The $\mathrm{n}_{2}$ coefficient they estimated for the modified area was approximate 5 times lower than the one they measured for the bulk, in very good agreement with the findings of this investigation. Moreover, it has been seen in studies on waveguides inscribed inside in fused silica substrates [19] that the ULI technique can cause changes to the nonlinear refractive index. It was shown that the nonlinear refractive indices of the waveguides were up to four times lower than that of bulk fused silica with their magnitude being very dependent on the inscription parameters.

Finally, it is worth mentioning that the CW cross-to-bar coupling ratio of about $60 \%$ for the two couplers of Fig. 2 is the highest we could achieve. This coupling ratio would be unlikely to reach $100 \%$ regardless of the sampling of the interaction length. This behavior is characteristic of differences in the propagation constants of the two waveguides forming the directional couplers [20], arising mainly from the fabrication procedure. In previous work by our group we showed that refractive index variations induced by ULI can extend beyond the geometrical structure of the waveguides up to a distance of $\sim 16 \mu \mathrm{m}$ [11]. Therefore, in the case of the directional couplers used in this work, where the core-to-core separation was chosen to be $7.5 \mu \mathrm{m}$, the optical properties (e.g. mode index) of the first waveguide for each coupler were directly affected by the inscription of the second 
waveguide and vice versa leading to different propagation constants for the two waveguides. A possible solution to this problem would be to choose core-to-core separations bigger than $\sim 20 \mu \mathrm{m}$. However, in this case the coupling coefficient would be greatly decreased and would therefore require very long interaction lengths for the maximum amount of power to transfer to the cross waveguide, which would cause other undesirable effects such as intermodal dispersion [21], completely jeopardizing the function of the directional couplers as optical switches. Other possible sources of the mismatch in the propagation constants of the two waveguides might be the drifts in laser power or temperature variations in the lab during the inscription process, where power fluctuations in the orders of only a few milliwatts or temperature variations as small as $\sim \pm 1^{\circ} \mathrm{C}$ might be sufficient to change the ULI parameters over the course of the experiment.

\section{CONCLUSION}

To summarize, we have investigated the nonlinear refraction properties of fs-laser inscribed structures. We have demonstrated ultrafast all-optical switching in nonlinear directional couplers in GLS at $1550 \mathrm{~nm}$ and by making use of the switching parameters we calculated the nonlinear refractive indices of the single-mode waveguides forming the directional couplers. It was found that the nonlinear refractive index of the modified, by the laser, area is reduced by the inscription process by about 4-5 times comparing to that of the bulk. We believe that our studies will contribute towards better understanding the nonlinear properties of ULI fabricated structures which will open the way for the optimum design of ultrafast laser inscribed integrated nonlinear optical devices. Finally, these studies are even more important in the case of chalcogenide glasses where their high Kerr nonlinearities make them very attractive candidates for such devices.

Funding. UK Engineering and Physical Sciences Research Council (EPSRC) (EP/G030227/1, EP/M015130/1). We have no underpinning data that needs archival storage within the content of this work.

Acknowledgment. The work of G Demetriou was supported by a James Watt scholarship from Heriot-Watt University.

\section{References}

1. B. J. Eggleton, B. Luther-Davies, and K. Richardson, "Chalcogenide photonics," Nat Photon 5, 141-148 (2011).

2. K. S. Bindra, H. T. Bookey, A. K. Kar, B. S. Wherrett, X. Liu, and A. Jha, "Nonlinear optical properties of chalcogenide glasses: Observation of multiphoton absorption," Applied Physics Letters 79, 1939-1941 (2001).

3. P. Bastock, C. Craig, K. Khan, E. Weatherby, J. Yao, and D. W. Hewak, "Properties of Gallium Lanthanum Sulphide Glass," in CLEO: 2015, OSA Technical Digest (online) (Optical Society of America, 2015), STh1G.1.

4. D. B. D.W.Hewak, R.J.Curry, G.R.Elliott, C.C.Huang, M.Hughes, K.Knight, A.K.Mairaj, M.N.Petrovich, R.Simpson, C.Sproat, "Chalcogenide glasses for photonics device applications," in Photonic glasses and glass-ceramics G. S. Murugan, ed. (2010), pp. 29-102.

5. K. M. Davis, K. Miura, N. Sugimoto, and K. Hirao, "Writing waveguides in glass with a femtosecond laser," Opt. Lett. 21, 1729-1731 (1996).

6. D. Choudhury, J. R. Macdonald, and A. K. Kar, "Ultrafast laser inscription: perspectives on future integrated applications," Laser \& Photonics Reviews 8, 827-846 (2014).

7. R. R. Thomson, H. T. Bookey, N. D. Psaila, A. Fender, S. Campbell, W. N. MacPherson, J. S. Barton, D. T. Reid, and A. K. Kar, "Ultrafast-laser inscription of a three dimensional fan-out device for multicore fiber coupling applications," Opt. Express 15, 11691-11697 (2007).
8. A. Ródenas, G. Martin, B. Arezki, N. Psaila, G. Jose, A. Jha, L. Labadie, P. Kern, A. Kar, and R. Thomson, "Three-dimensional mid-infrared photonic circuits in chalcogenide glass," Opt. Lett. 37, 392-394 (2012).

9. J. McCarthy, H. Bookey, S. Beecher, R. Lamb, I. Elder, and A. K. Kar, "Spectrally tailored mid-infrared super-continuum generation in a buried waveguide spanning $1750 \mathrm{~nm}$ to $5000 \mathrm{~nm}$ for atmospheric transmission, "Applied Physics Letters 103, 151103 (2013).

10. J. E. McCarthy, H. T. Bookey, N. D. Psaila, R. R. Thomson, and A. K. Kar, "Midinfrared spectral broadening in an ultrafast laser inscribed gallium lanthanum sulphide waveguide," Opt. Express 20, 1545-1551 (2012).

11. G. Demetriou, J.-P. Bérubé, R. Vallée, Y. Messaddeq, C. R. Petersen, D. Jain, O. Bang, C. Craig, D. W. Hewak, and A. K. Kar, "Refractive index and dispersion control of ultrafast laser inscribed waveguides in gallium lanthanum sulphide for near and mid-infrared applications," Opt. Express 24, 6350-6358 (2016).

12. M. Sheik-Bahae, A. A. Said, T. H. Wei, D. J. Hagan, and E. W. V. Stryland, "Sensitive measurement of optical nonlinearities using a single beam," IEEE Journal of Quantum Electronics 26, 760-769 (1990).

13. Y. Nasu, M. Kohtoku, and Y. Hibino, "Low-loss waveguides written with a femtosecond laser for flexible interconnection in a planar light-wave circuit," Opt. Lett. 30, 723-725 (2005).

14. R. Mary, D. Choudhury, and A. K. Kar, "Applications of Fiber Lasers for the Development of Compact Photonic Devices," IEEE Journal of Selected Topics in Quantum Electronics 20, 72-84 (2014).

15. A. Arriola, S. Mukherjee, D. Choudhury, L. Labadie, and R. R. Thomson, "Ultrafast laser inscription of mid-IR directional couplers for stellar interferometry," Opt. Lett. 39, 4820-4822 (2014).

16. R. W. Boyd, "Chapter 7 - Processes Resulting from the Intensity-Dependent Refractive Index," in Nonlinear Optics (Third Edition) (Academic Press, Burlington, 2008), pp. 329-390.

17. V. Mizrahi, M. A. Saifi, M. J. Andrejco, K. W. DeLong, and G. I. Stegeman, "Twophoton absorption as a limitation to all-optical switching," Opt. Lett. 14, 11401142 (1989).

18. J. S. Aitchison, A. H. Kean, C. N. Ironside, A. Villeneuve, and G. I. Stegeman, "Ultrafast all-optical switching in $\mathrm{Al}_{0.18} \mathrm{Ga}_{0.82}$ As directional coupler in $1.55 \mu \mathrm{m}$ spectral region," Electronics Letters 27, 1709-1710 (1991).

19. D. Blömer, A. Szameit, F. Dreisow, T. Schreiber, S. Nolte, and A. Tünnermann, "Nonlinear refractive index of fs-laser-written waveguides in fused silica," Opt. Express 14, 2151-2157 (2006).

20. S. M. Eaton, W. Chen, L. Zhang, H. Zhang, R. lyer, J. S. Aitchison, and P. R. Herman, "Telecom-Band Directional Coupler Written With Femtosecond Fiber Laser," IEEE Photonics Technology Letters 18, 2174-2176 (2006).

21. K. S. Chiang, "Propagation of short optical pulses in directional couplers with Kerr nonlinearity," J. Opt. Soc. Am. B 14, 1437-1443 (1997). 